\title{
Evaluación de las prácticas sanitarias que ejecutan algunos hatos ganaderos de la subregión Montes de María (Sucre, Colombia)
}

\author{
ARTÍCULO DE \\ INVESTIGACIÓN \\ María José Contreras-Gómez $\mathbb{D}^{1}{ }^{1}$, Caty Milena Martínez-Bravo (iD ${ }^{2}$, Libardo Enrique \\ Caraballo-Blanco (iD ${ }^{3}$ \\ 1 Bióloga. Joven investigador, Corporación Universitaria del Caribe (CECAR). \\ Sincelejo, Colombia. \\ 2 MSc. Directora del núcleo Problemas Asociados a la Producción Bovina, \\ Corporación Universitaria del Caribe (CECAR). Sincelejo, Colombia. \\ 3 Biólogo. MSc en Biotecnología, Universidad de Sucre. Sincelejo, Colombia.
}

\section{mallajose1309@gmail.com}

Recibido: 14 de Julio de 2016 y aprobado: 2 de Junio de 2017, Actualizado: Septiembre 3 de 2017

DOI: 10.17151/vetzo.2017.11.2.1

RESUMEN: La salud animal es uno de los aspectos más importantes dentro de los procesos de producción, y de mayor relevancia en el sector ganadero, donde las pérdidas generadas por la presencia de enfermedades en los hatos es un problema tanto económico como sanitario, a causa de las repercusiones en la salud pública. En este trabajo se pretendió conocer, por comunicación personal de los propios dueños de ganado, las principales enfermedades que afectan a los bovinos de la subregión Montes de María. Para su desarrollo se aplicó una encuesta evaluativa a los propietarios de hatos de la subregión y se revisaron los datos reportados por diferentes entidades gubernamentales. Se encontró que el 31,4\% (97/309) de los predios encuestados reportaron enfermedades, donde las más comunes resultaron ser la estomatitis vesicular bovina (EVB; 9,1\%), seguida de anaplasmosis (7,1\%) y enfermedades causadas por Clostridium (5,8\%). Al realizar el análisis estadístico, se encontró que no poseer un sistema de manejo de residuos sólidos podría llegar a considerarse un factor de riesgo, especialmente para enfermedades que involucren esta vía como fuente de dispersión del agente etiológico $(\mathrm{P}=0,02)$, al igual que desconocer y omitir la práctica de los programas de control y prevención de enfermedades $(\mathrm{P}=0,018$ y $\mathrm{P}=0,021)$. Por lo anterior se concluye que, pese a existir programas de control de enfermedades, estas siguen afectando a los bovinos principalmente a los pequeños productores o a quienes tienen ganado para uso propio.

Palabras clave: sanidad animal, bovinos, enfermedades. 


\title{
Evaluation of sanitary practices carried out by some cattle herds in the Montes de Maria sub-region (Sucre, Colombia)
}

\begin{abstract}
Animal health is one of the aspects most important fact in production processes, more in the livestock sector where losses generated by the presence of disease in herds is both economic and health problem because everything related is risk. In this paper, it was intended to know by personal communication of the owners of cattle, the main diseases affecting cattle in the sub-region Montes de Maria. An evaluative survey was applied to herd owners in the sub-region and the data reported by different governmental entities were reviewed. It was found that $31.4 \%$ (97/309) of surveyed reported illnesses land where the most common proved bovine vesicular stomatitis (EVB) 9.1\%, and anaplasmosis 7.1\% followed by diseases caused by Clostridium 5.8\%. Likewise, to performing statistical analysis, it was found that not having a solid waste management system, could be considered a risk factor, especially for diseases involving this pathway as a source of dispersion of the etiological agent $(\mathrm{P}=0.02)$, as well as no put into practice and ignore control programs and disease prevention ( $\mathrm{P}=0.018$ and $\mathrm{P}=0.021$ ). Therefore, it is concluded that despite the existence of disease control programs, these cattle continue to affect mainly small producers or have earned for own use.
\end{abstract}

Key words: animal health, cattle, diseases.

\section{Introducción}

La ganadería es una actividad económica que se lleva a cabo en muchos países del mundo. En Colombia, debido a su aporte a nivel social, económico y de generación de empleo, es de gran importancia en el desarrollo del sector pecuario (Goez-Carrascal, 2010).

En Colombia, la población bovina se distribuye en 494.402 predios y está constituida aproximadamente por 22’555.549 animales, de los cuales el 3,82\% corresponde a Sucre (ICA, 2016). El departamento de Sucre es uno de los de mayor vocación ganadera en el país, con una explotación ganadera de 862.008 cabezas para el año 2016, que representan el mayor aporte al PIB del departamento (21,1\%) (Osorio-Díaz, 2009).

La salud, el bienestar y la bioseguridad de los animales en el sector ganadero son aspectos de vital importancia, debido a que influyen directamente en la producción, por tal razón mantener la higiene de las instalaciones y practicar algunas medidas profilácticas como la vacunación, son indispensables para prevenir enfermedades en los animales y en el personal que se encuentre a cargo de los mismos, conllevando a evitar pérdidas económicas para los productores (Sánchez \& Cordero, 2011). En Colombia, en un estudio realizado en el departamento de Caldas se encontró que el $70 \%$ de los sistemas productivos fueron clasificados como de ceba extensiva, y el restante $30 \%$ de doble propósito, levante y cría. Los requisitos sanitarios para las instalaciones se encontraron en un nivel aceptable de implementación (74 al 96\%); la adopción de 
normas de bienestar animal (60 al 92\%), de sanidad animal y bioseguridad (15 al 74\%), y almacenamiento (bodegas limpias y ordenadas, separadas y cerradas, separación de plaguicidas y fertilizantes, control de temperatura y humedad) (23 al 59\%), en un nivel medio; siendo deficiente el plan de saneamiento ( 4 al 35\%) y el componente de personal (11 al 26\%), es decir, que no contaban con capacitación en bioseguridad y oficio, seguridad social y tener la dotación apropiada para el trabajo en los hatos (Romero \& Sánchez, 2011).

Conociendo la importancia de la ganadería bovina a nivel nacional y en el departamento de Sucre, así como la existencia de programas y normas referentes a la salud animal, y que además, se presentan problemas relacionados con enfermedades en los animales que repercuten inminentemente en la producción ganadera, el objetivo de este trabajo fue evaluar los aspectos sanitarios de los hatos ganaderos en la subregión Montes de María del departamento de Sucre, con el fin de conocer cuál es la ocurrencia de enfermedades con mayor prevalencia en el ganado y los factores asociados con su presentación.

\section{Materiales y Métodos}

\section{Área de estudio y tipo de estudio}

El área de estudio correspondió a los municipios ubicados en la subregión Montes de María del departamento de Sucre, la cual se ubica en la zona centro del departamento y abarca a los municipios de Sincelejo, Chalán, Ovejas, Morroa y Colosó (Figura 1). Su economía se basa en la producción agropecuaria, con predominio de la ganadería bovina y cultivos de arroz, maíz, plátano, yuca, ñame, tabaco, aguacate y café (Aguilera-Díaz, 2013).

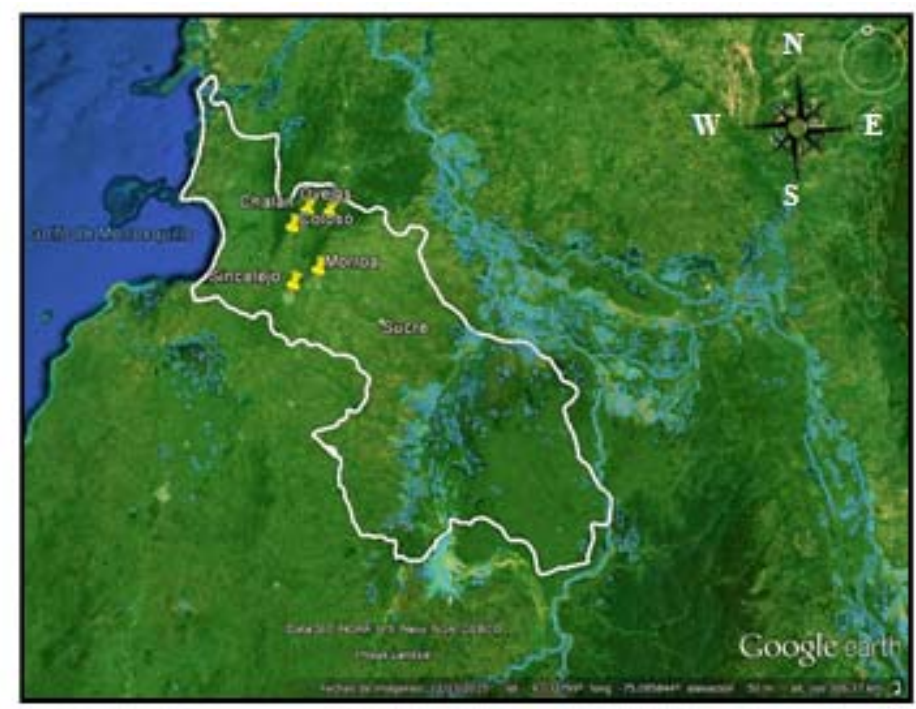

Fuente: Google earth, 2016.

Figura 1. Area de estudio, departamento de Sucre, subregión Montes de María. Municipios: Sincelejo, Morroa, Chalán, Colosó y Ovejas. 
Se realizó un estudio descriptivo correlacional, donde se tuvo en cuenta la relación del estado sanitario del hato ganadero y los factores de riesgo predisponentes a la aparición de enfermedades bovinas. Los datos fueron obtenidos en las fincas, mediante las encuestas evaluativas realizadas a propietarios y/o encargados de las mismas.

\section{Revisión de los registros de notificación de las entidades gubernamentales}

Se revisaron las bases de datos del Instituto Colombiano Agropecuario (ICA) y de la Federación Colombiana de Ganaderos (FEDEGÁN), para obtener los reportes de las enfermedades notificadas a partir del año 2012, tomando los correspondientes a la subregión Montes de María. Al estar dispersos, los datos fueron resumidos y organizados.

\section{Selección de los predios}

Se tomaron los registros de hatos registrados en las entidades gubernamentales (ICAFEDEGÁN) que correspondían a los municipios de la subregión Montes de María para ser involucradas en el estudio. Se encontraron en total 1.563 predios ubicados en la zona. El tamaño de la muestra se calculó con base a la población (1.563 fincas), con un error del 5\% y un nivel de confianza del 95\%.

Basado en este cálculo, se obtuvo un tamaño de muestra de 309 predios, donde se realizaron las encuestas, y dicho tamaño de la muestra fue distribuido entre los cinco municipios de la subregión Montes de María en proporción al número de fincas existentes en cada uno de ellos (Tabla 1). Sin embargo, durante el periodo de muestreo el número de encuestas aplicadas por municipio varió un poco respecto a los valores calculados.

Tabla 1. Número de fincas a encuestar por municipio según el cálculo de proporciones

\begin{tabular}{lc}
\hline Municipio & \# de muestras \\
\hline Chalán & 29 \\
Colosó & 28 \\
Ovejas & 95 \\
Sincelejo & 106 \\
Morroa & 51 \\
\hline Total & 309 \\
\hline
\end{tabular}

\section{Recolección de la información}

Se elaboró un instrumento siguiendo los lineamientos de la Resolución ICA 002341 de 2007 (ICA, 2007), enfocándose en la evaluación del estado sanitario de los predios, el cual fue aplicado en las fincas (Figura 2), con el consentimiento de los propietarios y acompañamiento de un vacunador de FEDEGÁN. 


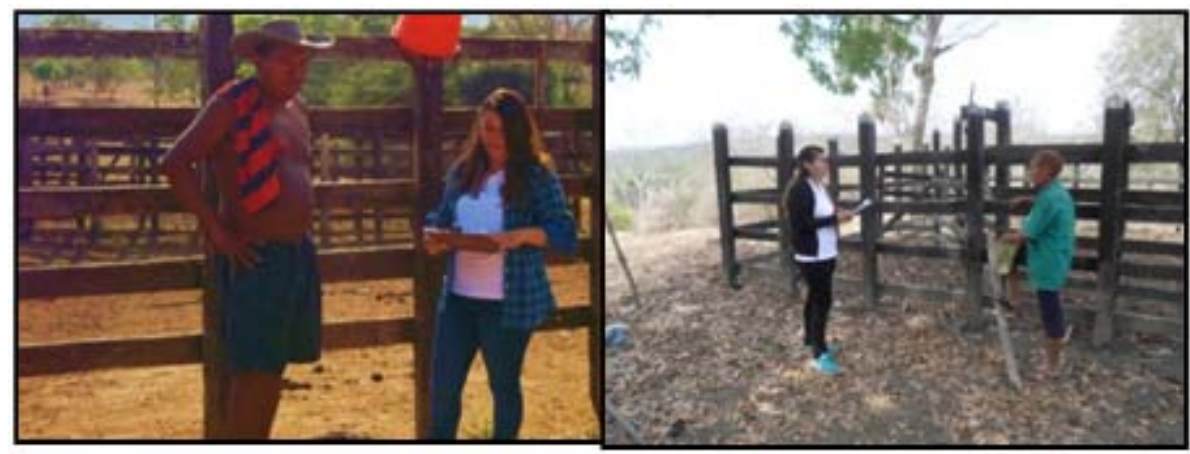

Figura 2. Recolección de los datos mediante encuetas realizadas a los dueños o encargados de las fincas (María José Contreras Gomez).

Para la identificación de las enfermedades de mayor notificación u ocurrencia en la zona de estudio, se consultaron los registros veterinarios de cada predio, se aplicó una encuesta evaluativa a los propietarios o encargados de las fincas y se consultaron los reportes de las entidades gubernamentales (ICA-FEDEGÁN). De esta manera, se evaluaron aspectos como: requisitos sanitarios para instalaciones y áreas, plan de saneamiento, tipo de alimentación, sanidad animal, bioseguridad, mortalidad y el tipo de sistema de producción, todo esto para evaluar la relación entre los factores y la presencia de enfermedades diagnosticadas por veterinarios o zootecnistas consultados cuando el ganado presenta algún cambio en su comportamiento (registros médicos no incluidos).

\section{Análisis estadístico}

Los datos obtenidos fueron tabulados en el programa Microsoft Excel 2010. Se realizó un análisis descriptivo utilizando los softwares estadísticos InfoStat (Di Rienzo et al., 2016) versión estudiantil y SPSS 22 (IBM, 2013), con las variables incluidas en el estudio. Posteriormente, se aplicaron análisis de correspondencia múltiple (ACM) (Lebart et al., 1995) para estudiar la asociación entre las condiciones sanitarias, los factores tales como: manejo de excretas, fuentes de agua, tipo de alimentación, manejo del ganado nuevo y mortalidad; tipo de sistema de producción (doble propósito, ceba, carne, leche) y los registros de presencia de enfermedades reportados por médicos veterinarios o zootecnistas que son consultados cuando los animales están enfermos.

\section{Resultados y Discusión}

Se consultaron las bases de datos del ICA y FEDEGÁN donde se llevan los registros de notificación de enfermedades, tomándose solo los que fueron notificados por municipios de la subregión Montes de María; teniendo en cuenta esto, se encontró que en el año 2012 se notificaron 38 casos de rabia silvestre, de los cuales 31 fueron positivos. Asimismo, se presentaron 3.962 casos de brucelosis confirmados (Osorio et al., 2013). Para 2013, se reportaron 13 casos de estomatitis vesicular y 47 de rabia silvestre, y un caso de aftosa que resultó negativo, comprobándose el funcionamiento del programa de erradicación de aftosa implementado en el departamento de Sucre (ICA, 2013). En 2014 se presentaron 4 casos de rabia silvestre y 14 casos de estomatitis 
vesicular, con lo que se infiere que los reportes de casos de las distintas enfermedades han disminuido, aunque se mantienen los reportes de rabia silvestre, y se infiere asociación con las condiciones del medio ambiente que favorecen la alta presencia de murciélagos hematófagos transmisores del virus de la rabia, los cuales encuentran en el ganado una buena fuente de ingesta de sangre (ICA, 2014).

Hasta el mes de agosto de 2015 se reportaron 6 casos de rabia y 5 de síndrome neurológico (encefalopatía espongiforme bovina), que aparece como una enfermedad reemergente, pero escasa en el departamento de Sucre y que puede deberse a la introducción de ganado bovino proveniente de otras regiones del país (ICA, 2015).

Hay que anotar que, en los registros revisados del ICA, las enfermedades estudiadas y monitoreadas son las declaradas como de control oficial, es decir aquellas que tienen lineamientos establecidos para su manejo y erradicación; y que están descritas en la Resolución 003714 del ICA. Sin embargo, existen enfermedades y síntomas de control no oficial (leptospirosis, neosporosis, campilobacteriosis, pasterelosis, mastitis, parasitarias, carenciales) que están presentes en todas las regiones de Colombia y tienen repercusiones en la producción, reproducción de los animales y en la estabilidad económica del negocio ganadero (Santos, 2014).

Después de haber revisado los reportes oficiales, se procedió a realizar las encuestas en las fincas para revisar de manera directa el estado sanitario de los predios. Inicialmente, en cuanto al tipo de explotación y el uso del ganado, se encontró un predominio de la ganadería extensiva $(96,12 \%)$ donde el ganado es utilizado en su mayoría para la producción de leche $(57 \%)$, la cual es obtenida de manera artesanal y para uso local principalmente, es decir, venta en el pueblo y alrededores, así como también para la elaboración de suero, queso y otros derivados lácteos que sirven de sustento a las familias, lo que muestra la baja tecnificación del sector ganadero, y el poco desarrollo empresarial y productivo.

En lo referente a los casos de enfermedades, se encontró que en el 31,39\% de los predios visitados, según la persona encargada del hato y registros médicos (datos no publicados), se habían presentado enfermedades (tabla 2).

Además, de las enfermedades indicadas por los encargados del hato, producidas de manera general por bacterias, parásitos y virus, se encontró que las más comunes en los predios encuestados, fueron la estomatitis vesicular bovina (EVB) 9,1\%, la anaplasmosis 7,1\% y enfermedades causadas por Clostridium 5,8\%, principalmente carbón sintomático.

Dentro de las enfermedades reportadas, la EVB fue una de las de mayor ocurrencia según lo reportado en las encuestas, enfermedad que no hace parte de los planes de control oficial del gobierno, y que afecta gravemente al ganado bovino, pero también tiende a afectar a los operarios del sistema de producción, lo que a futuro puede llegar a generar problemas de salud pública (APHIS, 2012). La presencia de esta enfermedad puede estar relacionada con una persistencia del agente causal entre distintas especies animales del departamento de Sucre que mantienen activo el ciclo de vida del virus, puesto que históricamente Sucre se ha señalado como uno de los departamentos con mayor incidencia de brotes de EVB (Trujillo-Bowen, 2009), además las condiciones medioambientales y la presencia de vectores de la enfermedad (como moscas del 
género Lutzonia shanoni y algunos simúlidos) pueden estar jugando un rol importante en la incidencia de la EVB en el departamento, en específico, la subregión Montes de María (Trujillo-Bowen, 2009).

Otra enfermedad reportada por los dueños u encargados de los hatos, y que causa grandes pérdidas económicas a nivel mundial, es la anaplasmosis (Corona et al., 2004), que según los reportes del ICA presenta una incidencia nacional de 4 por 100 y que para el año 2012 causó pérdidas económicas por encima de los 1000 USD (Díaz et al., 2012).

$\mathrm{Al}$ analizar los posibles factores asociados a la presencia de enfermedades, se encontró que el $84,5 \%$ de los bovinos de los predios no consumen agua potable, es decir proveniente del acueducto. Sin embargo, al hacer el análisis por ji-cuadrado $(\mathrm{P}=0,131)$ no se encontró significancia entra estas dos variables. Aun así, hay que tener en cuenta que la calidad del agua disponible para los animales es tan importante como la cantidad. El agua que beben los bovinos debe ser limpia, inodora, incolora e insípida, por lo que el consumo de agua de baja calidad, es decir que no cumpla las condiciones antes mencionadas, determina pérdida de peso en los animales, falta de apetito, trastornos digestivos, reducción en la producción láctea, alteración en la reproducción, enfermedades y muerte en los casos más extremos (Cseh, 2003) a causa de bacterias, virus y parásitos que son comunes en aguas procedentes de pozos y reservorios que colectan aguas residuales, o a las que el ganado tiene acceso, facilitando una diseminación rápida de alguna enfermedad en el hato (Olkowski, 2009).

En cuanto a las variables asociadas al plan de saneamiento, se encontró asociación entre el sistema de manejo de residuos sólidos y el reporte de presencia de enfermedades $(\mathrm{P}=0,049)$. Seguido a esto, se realizó una regresión multinomial (Hosmer \& Lemeshow, 1989) enfrentando todas las variables de plan de saneamiento frente a la presencia de enfermedades, con lo que se constató que no tener un sistema de manejo de residuos es un factor que se asocia a la presencia de enfermedades (Tabla 3).

Lo anterior puede estar asociado a que, en el área de estudio, la mayoría de las fincas (212/309) no contaban con un sistema de tratamiento de las excretas de los bovinos, puesto que las dejan en el establo o las recogen y apilan a un lado de este hasta que llueva y sean arrastradas por las corrientes, lo que lleva a sospechar que, al no tener un sistema de manejo de dichos residuos, se mantenga un ciclo infectivo de contaminación entre los animales del predio, debido a la acumulación del material fecal de los bovinos que representa un riesgo ocupacional y de diseminación de microorganismos comoCryptosporidium, Giardia, Coxiella, Leptospira, Mycobacterium, Salmonella, E. coli, Listeria monocytogenes, entre otros, lo cual afecta la salud animal y por ende lo que se deriva de esto (Cediel \& Villamil, 2004; Klein et al., 2010).

En cuanto a la sanidad animal y bioseguridad, se encontró que desconocer los programas de control de enfermedades $(\mathrm{P}=0,018)$ y no poner en práctica dichos programas $(\mathrm{P}=0,021)$ presentan relación estadística con la presencia de enfermedades en los predios, lo cual denota riesgo para la cría del ganado y de todo lo que se deriva de este proceso, aunque esto se debe posiblemente a la relación costo-beneficio que puede llegar a generar la implementación de este tipo de programas. Además, hay que tener en cuenta que las creencias culturales, la falta de conocimiento y la ausencia de presiones sociales y económicas por parte de los consumidores, son factores que inciden en la baja 
adopción de estos programas, sumado a que la mayoría de los predios involucrados en el estudio pertenecían a medianos y pequeños productores (Romero \& Sánchez, 2011).

Finalmente, en cuanto a la mortalidad y a la ocurrencia de abortos, se encontró asociación entre la ocurrencia de aborto $(\mathrm{P}=0,038)$ y la presencia de enfermedades. Así mismo, para el caso de muertes y presencia de enfermedades, dicha relación fue altamente significativa (Tabla 4).

Lo anterior, resalta la problemática sanitaria que ha venido afectando a los predios ganaderos de la subregión Montes de María (Sucre), así como la ocurrencia de abortos, los cuales en muchos casos son inducidos por la infección con microrganismos patógenas que afectan a la vaca gestante, la debilitan, e inducen a la pérdida del feto, principalmente por agentes causantes de enfermedades como la brucelosis, la leptospirosis, la rinotraqueitis infecciosa bovina, entre otras; estos factores de mortalidad afectan de manera directa la productividad, al disminuir el inventario ganadero de los hatos.

Todo esto sugiere que hay que tomar medidas de control que incluyan las enfermedades que están dentro de las no notificables, puesto que están afectando a los predios ganaderos del departamento de Sucre de manera silenciosa, lo que agrava la situación sanitaria de los pequeños ganaderos principalmente.

Tabla 2. Proporción de ocurrencia de enfermedades en los predios visitados de los distintos municipios de la subregión Montes de María (Sucre)

\begin{tabular}{ccccccc}
\hline \multirow{2}{*}{ Ocurrió enfermedad } & \multicolumn{5}{c}{ Municipio } & \multirow{2}{*}{ Total } \\
\cline { 2 - 6 } & Chalán & Colosó & Moros & Ovejas & Sincéejo \\
\hline No & 27 & 20 & 30 & 70 & 65 & 212 \\
Sí & 5 & 9 & 19 & 30 & 34 & 97 \\
\hline Total fincas visitadas & 32 & 29 & 49 & 100 & 99 & 309 \\
\hline
\end{tabular}


Tabla 3. Regresión logística presencia de enfermedades - plan de saneamiento

\begin{tabular}{|c|c|c|c|c|c|c|c|c|c|}
\hline \multirow{2}{*}{\multicolumn{2}{|c|}{ Enfermedgd }} & \multirow[t]{2}{*}{ B } & \multirow[t]{2}{*}{$\begin{array}{l}\text { Error } \\
\text { eständar }\end{array}$} & \multirow[t]{2}{*}{ Wald } & \multirow[t]{2}{*}{ gा } & \multirow[t]{2}{*}{ Sig. } & \multirow[t]{2}{*}{$\operatorname{Exp}(B)$} & \multicolumn{2}{|c|}{$\begin{array}{c}95 \% \text { de intervalo de } \\
\text { confianza pars } \\
\text { Exp(B) }\end{array}$} \\
\hline & & & & & & & & $\begin{array}{l}\text { Limite } \\
\text { inferior }\end{array}$ & $\begin{array}{l}\text { Limite } \\
\text { superior }\end{array}$ \\
\hline \multirow[t]{9}{*}{ s } & Tnterceptacion & $-0,680$ & 0,343 & 3,929 & 1 & 0,047 & & & \\
\hline & [Cupglyd=no] & 0,517 & 0,299 & 2,995 & 1 & 0,084 & 1,677 & 0,934 & 3,012 \\
\hline & [Cupglyd=s] & $0^{-}$ & . & . & 0 & . & . & . & . \\
\hline & [PosestP=no] & $-0,412$ & 0,384 & 1,150 & 1 & 0,283 & 0,652 & 0,312 & 1,407 \\
\hline & [Posestr=s] & $0^{-}$ & . & . & 0 & . & . & . & . \\
\hline & [PosesTMRS=no] & $-0,673$ & 0,289 & 5,441 & 1 & 0,020 & 0.510 & 0,290 & 0,898 \\
\hline & [PoseSIMRS=s!] & $0^{-}$ & $=$ & . & 0 & 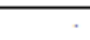 & . & . & \\
\hline & [Cuggusp=no] & 0,148 & 0,349 & 0,181 & 1 & 0,671 & 1,160 & 0,585 & 2,299 \\
\hline & [Cugguap=s!] & & & & & 0 & 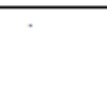 & 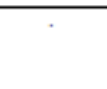 & . \\
\hline \multicolumn{10}{|c|}{ gl: grados de libertad. sig: significancia. B: coeficiente. Exp (B): ries go. Wald: valor chi. Cupglyd: } \\
\hline \multicolumn{10}{|c|}{$\begin{array}{l}\text { Cuentan con un Programa limpieza y desinfección. PoseSIP: Poseen sistemas de control integrado } \\
\text { de plagas. PoseSMRS: Poseen sistemas de manejo de residuos sólidos. Cuaguap: Cuentan con }\end{array}$} \\
\hline
\end{tabular}

Tabla 4. Análisis ji-cuadrado presencia de enfermedades en relación a la ocurrencia de abortos y muertes de bovinos en los predios

\begin{tabular}{ccccccc}
\hline Enfermedades & \multicolumn{2}{c}{ Abortos } & & & \multicolumn{2}{c}{ Muertes } \\
& Si & No & P-valor & Si & No & P-valor \\
& & & & & & \\
\hline Si & 28 & 69 & 0,038 & 55 & 51 & 0,0001 \\
\hline No & 173 & 39 & & 42 & 161 & \\
\hline Total & 201 & 108 & & 97 & 212 & \\
\hline
\end{tabular}

\section{Conclusiones}

Las muertes ocurridas en los predios están relacionadas con la presencia de enfermedades, entre estas se encuentra la EVB, la cual es una de las enfermedades que más se presenta en los hatos ganaderos de la subregión Montes de María (Sucre). Además, cabe resaltar que tanto el manejo inadecuado de las excretas de los bovinos como el consumo de agua no apta para los animales (proveniente de pozos, jagüeyes), son factores predisponentes en la aparición de enfermedades del ganado en la subregión Montes de María. 


\section{Agradecimientos}

Se agradece al Programa de Formación de Capacidades en Ciencia, Tecnología, e Innovación en el Departamento de Sucre, Corporación Universitaria del Caribe (CECAR), por la financiación de este proyecto.

\section{Referencias bibliográficas}

- Aguilera-Díaz, M. Montes de María: Una subregión de economía campesina y empresarial. Cartagena, Colombia: Banco de la República, 2013. 93p.

- APHIS - Animal and Plant Health Inspection Service. Factsheet Veterinary Services: Vesicular Stomatitis, 2012. 2p. Disponible en: Link. Accesado en 05/01/2016.

- Cediel, N.; Villamil, L.C. Riesgo biológico ocupacional en la Medicina Veterinaria, área de intervención prioritaria. Revista Salud Pública, v.6, n.1, p.28-43, 2004.

- Corona, B.; Rodríguez, M.; Martínez, S. Anaplasmosis bovina (bovine anaplasmosis).Revista Electrónica de Veterinaria, v.7, n.4, p.1-27, 2004.

- Cseh, S. El agua y su importancia para los bóvidos. Dpto. Producción Animal INTA Balcarce. Sitio argentino web producción animal, 2003. p.1-4. Disponible en:Link Accesado en 05/01/2016

- Díaz, O.; Ortiz, J.; Reina, J. et al. Colombia, Sanidad Animal 2011. Colombia: Instituto Colombiano Agropecuario, 2012. 144p.

- Di Rienzo, J.A.; Casanoves, F.; Balzarini, M.G. et al. InfoStat versión 2016. Grupo InfoStat, FCA, Universidad Nacional de Córdoba, Argentina. 2016. Disponible en: LinkAccesado en 15/01/2016.

- Goez-Carrascal, M. Implementación de buenas prácticas ganaderas en la hacienda la María en el municipio de Puerto Berrío (Ant.). Caldas, Colombia: Corporación Universitaria Lasallista, 2010. 69p. (Informe de práctica para optar al título de Industrial Pecuaria).

- Hosmer, D.W.; Lemeshow, S. Applied Logistic Regression. New York: Wiley Interscience, 1989.

- IBM Corp. Released 2013. IBM SPSS Statistics for Windows, Version 22.0.Armonk, NY: IBM Corp., 2013.

- ICA - Instituto Colombiano Agropecuario. Resolución 002341, Condiciones sanitarias y de inocuidad en la producción primaria de ganado bovino y bufalino destinado al 
sacrificio para consumo. Boletín Oficial del Estado, n.46730. Bogotá, D.C., Colombia, 2007. 19p. Disponible en: Link Accesado en 22/09/2015.

- ICA - Instituto Colombiano Agropecuario. Boletines epidemiológicos mensuales, 2013. Disponible en: Link Accesado en 02/12/2015.

- ICA - Instituto Colombiano Agropecuario. Boletines epidemiológicos mensuales, 2014. Disponible en: Link Accesado en 02/12/2015.

- ICA - Instituto Colombiano Agropecuario. Boletines epidemiológicos, mensuales, 2015. Disponible en: Link Accesado en 05/12/2015.

- ICA - Instituto Colombiano Agropecuario. Censo Pecuario Nacional - 2016. Censo bovino en Colombia, 2016. Disponible en: Link Accesado en 04/07/2016.

- Klein, M.; Brown, L.; Van den Akker, B. et al. Monitoring bacterial indicators and pathogens in cattle feedlot waste by real-time PCR. Water Research, v.44, p.13811388, 2010.

- Lebart, L., Morineau, A., Piron, M. Statistique exploratoire multidimensionnelle. Paris: Dunod, 1995.

- Olkowski, A.A. Livestock Water Quality: A Field Guide for Cattle, Horses, Poultry and Swine. Minister of Agriculture and Agri-Food Canada, 2009. 181p.

- Osorio, F.J.; Patiño, A.; Linares, C. et al. Sanidad animal-2012. Bogotá, D.C., Colombia: Instituto Colombiano Agropecuario - ICA, 2013. 152p. Disponible en: LinkAccesado en 09/08/2015.

- Osorio-Díaz, Y.M. Evaluación del sistema de producción bovino cría en la finca Mundo Nuevo ubicada en el Golfo de Morrosquillo en el Departamento de Sucre. Sucre, Colombia. Universidad de Sucre, 2009. 67p. (Pregrado en la modalidad pasantía o proyección social, para optar el título de Zootecnista).

- Romero, M.; Sánchez, J. Evaluación de las buenas prácticas ganaderas en bovinos de carne en el centro de Caldas. Biosalud, v.10, n.1, p.52-60, 2011.

- Sánchez, T.; Cordero, J. Medidas de higiene y sanitarias en ganado bovino. Secretaría de Agricultura, Ganadería, Desarrollo Rural, Pesca y Alimentación (México) Sagarpa, v.9, n.1. 2011. Disponible en: Link Accesado en 07/06/2016.

- Santos, S. 14 enfermedades sin control oficial atacan al ganado en Colombia. Reporte contexto ganadero, 2014. Disponible en: Link Accesado en 02/12/2015.

- Trujillo-Bowen, L.M. Antecedentes y avances en aspectos de epidemiologia, diagnóstico y control de la estomatitis vesicular. Bogotá, D.C., Colombia: Pontificia 
Universidad Javeriana, 2009. 73p. Tesis (Pregrado en Microbiología Agrícola y Veterinaria).

Cómo citar: Contreras-Gómez, M.J.; Martínez-Bravo, C.M.; Caraballo-Blanco, L.E. Evaluación de las prácticas sanitarias que ejecutan algunos hatos ganaderos de la subregión Montes de María (Sucre, Colombia). Revista Veterinaria y Zootecnia, v.11, n.2, p.1 - 12, 2017. DOI: 10.17151/vetzo.2017.11.2.1

Esta obra está bajo una Licencia de Creative Commons Reconocimiento CC BY

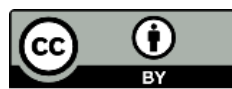

